\section{Papel da vitamina A na prevenção do estresse oxidativo em recém-nascidos}

\section{Vitamin A role preventing oxidative stress in newborns}

\author{
Mirian Martins Gomes 1 \\ Cláudia Saunders 2 \\ Elizabeth Accioly 3
}

\section{Abstract}

Vitamin A is recognized as an essential trace element in phases of intense growth and development such as pregnancy and childhood. The influence of this trace element is recognized in different metabolic processes and is currently emphasized as an antioxidant. Pro-vitamin forms (carotenoids) are outstanding. Anti-oxidant action is very valuable at birth for this is a period of intense free radical production by newborns caused by exposure to high oxygen concentrations. Placental transference of these nutrients occurs predominantly during the last trimester of pregnancy and is dependent on maternal serum concentration. Premature newborns are more vulnerable to the consequences of this stress due to low reserves and immaturity of the other anti-oxidant systems. Controversies remain related to the beneficial effect of supplementation. Therefore, prenatal nutrition education plays a crucial role in Vitamin A deficiency prevention and related consequences. This review intends to gather literature related to the issue published between 1990 and 2003 and referred by the MEDLINE and LILAC databases.

Key words Vitamin A, Carotenoids, Infant, newborn, Prematurity, Oxidative stress

\section{Resumo}

A vitamina A é um micronutriente essencial ao ser humano, sobretudo nos momentos de intenso crescimento e desenvolvimento, como a gestação e a infância. Além da atuação dessa vitamina em diversos processos metabólicos, atualmente têm-se destacado o efeito antioxidante desempenhado principalmente pelas formas pró-vitamínicas (carotenóides). Essa função antioxidante da vitamina A é de grande importância no nascimento, período no qual o recémnascido produz grande quantidade de radicais livres em resposta à exposição a elevadas concentrações de oxigênio. Devido às baixas reservas dessa vitamina, cuja transferência trans-placentária ocorre principalmente no terceiro trimestre de gestação e devido à imaturidade dos demais sistemas antioxidantes, os recém-nascidos prematuros são os mais vulneráveis aos efeitos do estresse decorrente do nascimento. No entanto, ainda existem controvérsias quanto aos benefícios da suplementação da vitamina A no período gestacional, com o objetivo de prevenir a deficiência de vitamina A e suas conseqüências. O presente trabalho tem por objetivo reunir artigos referentes ao tema e publicados em periódicos indexados nas bases de dados MEDLINE e LILACS, no período de 1990 e 2003.

Palavras-chave Vitamina A, Carotenóides, Recémnascido, Prematuridade, Estresse oxidativo. 


\section{Introdução}

A vitamina A (VA) foi a primeira vitamina lipossolúvel a ser reconhecida, fato ocorrido em 1913,1 e desde então numerosos estudos vêm sendo conduzidos a fim de elucidar as funções desse nutriente. Inicialmente, o papel da VA era apenas reconhecido na fisiologia do sistema visual. ${ }^{2}$ Posteriormente, as consequiências da deficiência de vitamina A (DVA) sobre a função imune, assim como seu reflexo nas taxas de morbimortalidade, em especial no grupo materno-infantil, foram estabelecidas. ${ }^{3-9}$

A VA atua no metabolismo intermediário, na síntese de ácido ribonucléico (RNA) e proteínas, enzimas, globulinas, glicoproteínas, queratina, na permeabilidade celular e nos metabolismos da hemoglobina e do zinco. ${ }^{1}$ Blaner, 10 revisando os mecanismos moleculares da ação da vitamina $\mathrm{A}$, sugere que esse micronutriente participa dos receptores nucleares e seus ativadores, que regulam a expressão de genes relacionados ao metabolismo da triiodo-tironina, estrogênio, progesterona, cortisol, aldosterona, testosterona, vitamina $\mathrm{D}$, colesterol e ácidos graxos. Dessa forma, a carência desse nutriente poderá interferir, por exemplo, na reprodução, no crescimento e desenvolvimento infantil.11-14

Mais recentemente, a ação antioxidante da VA tem ganhado destaque, 15,16 fato que impulsionou a investigação de seus precursores, particularmente os carotenóides, os quais anteriormente eram reconhecidos apenas por sua atividade pró-vitamínica, 17 e que atualmente são considerados como importantes antioxidantes. $15,16,18-21$

Os estudos recentes têm enfocado especialmente à importância de níveis antioxidantes adequados no momento do parto, considerada uma condição de estresse oxidativo.15,22,23 (Quais os efeitos do estresse oxidativo). Nos prematuros, esse momento é ainda mais preocupante devido à imaturidade dos sistemas antioxidantes e reservas inadequadas de nutrientes que agem como tal. ${ }^{24}$ Os radicais livres estão associados às enfermidades causadas pelo estresse oxidativo 25,26 e, nesse particular, ganham destaque os danos causados ao sistema respiratório.27-29

O presente trabalho tem por objetivo reunir informações disponíveis na literatura acerca das funções da VA e seus precursores, assim como das conseqüências de sua deficiência para a saúde do recém-nascido (RN) no período pós-natal imediato, com enfoque especial aos efeitos antioxidantes para o referido grupo. Para tal foram pesquisados artigos referentes ao tema e publicados em periódicos indexados nas bases de dados MEDLINE e LILACS no período de 1990 a 2003. Os termos de acesso foram: "Newborn",
"Premature infants", "Vitamin A", "Carotenoids", "Antioxidants;" "Free radicals", "Oxidative stress", "Oxigen supply".

\section{Vitamina A: nutriente essencial}

Vitamina A é a expressão genérica usada para descrever o retinol e todos os carotenóides dietéticos que têm atividade biológica de transretinol. A VA natural usualmente se apresenta na forma de ésteres de retinil de cadeia longa. As formas metabolicamente ativas incluem os correspondentes aldeído (retinal) e ácido (ácido retinóico). Já o termo retinóides refere-se ao retinol, seus metabólitos e análogos sintéticos que têm estrutura similar.1

Existem mais de 600 formas de carotenóides na natureza. Desses, aproximadamente 20 têm atividade de pró-vitamina A, porém dados de composição em alimentos são disponíveis apenas para três $(\alpha-$ caroteno, $\beta$-caroteno e $\beta$-criptoxantina) sendo esses os mais importantes precursores da VA em humanos. 1,30

A VA foi chamada de retinol em referência a sua função específica na retina . A importância da DVA, até a segunda metade da década dos anos 80, era associada aos sinais clínicos oftalmicos- xeroftalmia, que evolui da cegueira noturna até a cegueira nutricional irreversível. 2 A partir da segunda metade dessa década, evidências apontaram que a carência subclínica, sem sinais como xeroftalmia (mancha de Bitot e ceratomalácia), também poderia ter impacto negativo na morbidade e mortalidade de RN, préescolares, mulheres em idade fértil, gestantes, puérperas e nutrizes, os grupos tradicionalmente considerados "de risco nutricional".4-9,31

Estima-se que 150 milhões de mulheres e crianças no mundo apresentam a DVA, 4,4 milhões de pré-escolares apresentam xeroftalmia e 6,2 milhões de mulheres desenvolvam cegueira noturna durante a gestação. 32 Aproximadamente 500.000 crianças evoluem para a cegueira nutricional irreversível anualmente. Mais da metade dessas morrem poucos meses após tornarem-se cegas.7,33,34

Os benefícios de um adequado estado nutricional de VA sobre a saúde do grupo materno infantil podem ser atribuídos aos efeitos do retinol, que atua na manutenção da integridade epitelial e do sistema imunológico e, aos carotenóides, que têm ação antioxidante. 35 Já é reconhecido que um adequado estado nutricional de VA pode diminuir a severidade dos quadros infecciosos, 36,37 enquanto a deficiência pode aumentá-la, ${ }^{3}$ devido a prejuízos no sistema imunológico. 38 
A fim de reduzir os efeitos deletérios causados pela DVA, vários estudos têm sido conduzidos objetivando avaliar o impacto de sua suplementação em gestantes,4,31 lactantes 4,39-41 e RN.42-44 Existem, porém, muitas especulações quanto a melhor forma de administração (retinol e/ou carotenóides) e a dose capaz de produzir benefícios sem efeitos deletérios, sobretudo no que diz respeito às gestantes e RN. Dessa forma, foram estabelecidas doses máximas seguras a fim de evitar os efeitos deletérios da superdosagem de retinol, principalmente para o grupo materno infantil,45,46 conforme apresentado no Quadro 1.

\section{Carotenóides: efeito antioxidante}

Das diversas espécies de carotenóides, os cinco mais comumente encontrados no plasma humano são o $a$ caroteno, o ß-caroteno, a ß-criptoxantina, a luteína e o licopeno. ${ }^{47}$ Entre esses, o $\beta$-caroteno é o mais potente precursor de retinol $14,20,47$ e durante muito tempo apenas essa função foi estudada. ${ }^{17}$ Independente de sua atividade de pró-vitamina A os carotenóides são responsáveis por outros efeitos benéficos para a saúde humana. 47

Os carotenóides são importantes seqüestradores de radicais oxigênio singlet, tendo sido reconhecidos pela sua alta capacidade antioxidante15,16,18-21, interrompendo a geração de caratenóides reativos ao oxigênio ainda nas etapas iniciais de sua formação. ${ }^{17}$ Tal efeito também vem sendo atribuído mais recentemente ao próprio retinol, do qual alguns carotenóides são precursores.15,16 Uma única molécula de retinol ou $\beta$-caroteno é capaz de inati-

Quadro 1

Doses máximas seguras de retinol para tratamento e prevenção da xeroftalmia e prevenção da deficiência de vitamina A (DVA).

\begin{tabular}{|c|c|}
\hline Faixa etária & Dose máxima segura \\
\hline \multicolumn{2}{|c|}{ Tratamento e prevenção da xeroftalmia } \\
\hline \multicolumn{2}{|l|}{ Crianças } \\
\hline$<1$ ano & $100.000 \mathrm{UI}$ \\
\hline 1 ano & $200.000 \mathrm{UI}$ \\
\hline \multicolumn{2}{|l|}{ Gestantes } \\
\hline & $10.000 \mathrm{UI} / \mathrm{dia}$ \\
\hline & $25.000 \mathrm{UI} / \mathrm{semana}$ \\
\hline \multicolumn{2}{|l|}{ Nutrizes } \\
\hline Amamentando & 200.000 UI até oito semanas pós parto \\
\hline Sem amamentar & 200.000 UI até oito semanas pós parto \\
\hline \multicolumn{2}{|c|}{ Prevenção da DVA } \\
\hline \multicolumn{2}{|l|}{ Crianças } \\
\hline $6-11$ meses & $100.000 \mathrm{UI}$ a cada três a seis meses \\
\hline $1-5$ anos & 200.000 UI a cada três a seis meses \\
\hline Gestantes & $10.000 \mathrm{UI} / \mathrm{dia}$ \\
\hline Nutrizes & (dose única) \\
\hline Amamentando & 200.000 UI até oito semanas pós parto \\
\hline Sem amamentar & $200.000 \mathrm{UI}$ até seis semanas pós parto \\
\hline Mulheres em idade fértil & $10.000 \mathrm{UI} / \mathrm{dia}$ \\
\hline$(10-49$ anos $)$ & \\
\hline
\end{tabular}

IVACG=International Vitamin A Consultative Group; 1997; 1998.45,46 
var vários radicais oxigênio singlet antes de ser destruída. 16,48 O ß-caroteno é ainda reconhecido como varredor de radicais peroxil, especialmente em condições de baixa tensão de oxigênio. Contudo é possível que outros carotenóides também possuam essa atividade. 21

Embora inúmeros estudos epidemiológicos sugiram que os carotenóides têm funções biológicas relacionadas à prevenção de doenças crônicas, seu papel em RN tem merecido investigação. 19

Quando o consumo de carotenóides com atividade pró-vitamínica é baixo $(<1 \mathrm{mg})$, a maior parte destes é convertida a VA no intestino delgado. Contudo, com a ingestão de maiores quantidades, uma maior proporção de carotenóides absorvidos é encontrada no plasma e nos tecidos. $30 \mathrm{O}$ consumo de alimentos ricos em carotenóides poderia alterar a concentração de carotenóides maternos e, dessa forma, favorecer a formação de reservas do RN.19 Outra alternativa seria a suplementação de gestantes. $\mathrm{O}$ aumento dos estoques poderia reduzir o estresse oxidativo do RN resultante da exposição ao oxigênio no momento do parto e no período neonatal imediato. 24 Esse procedimento, a princípio, não traria conseqüências danosas para o grupo materno-infantil, visto que o $ß$-caroteno, assim como os outros carotenóides, não possuem efeito carcinogênico ou teratogênico e não causam hipervitaminose A.17,20

Recentemente tem sido discutido o fato de os metabólitos intermediários dos carotenóides, em especial do $ß$-caroteno, terem atividade própria e serem tóxicos. Em condições normais essa produção é relativamente baixa e não traz conseqüências. Porém há indícios de que em situações de suplementação com ß-caroteno sob condições de estresse oxidativo, haveria acúmulo desses metabólitos. A atuação desses e os possíveis efeitos nocivos ainda permanecem sob estudos. 49 De qualquer forma nos impõe cautela a indicação da suplementação de carotenóides para redução de danos causados pelo estresse oxidativo.

\section{Transferência de vitamina A durante a vida intra-uterina e a lactação}

A placenta parece exercer um rigoroso controle na transferência materno-fetal de VA, mantendo suprimento adequado para o feto apesar de uma ampla variação da VA materna circulante. 50,51

Em razão desse mecanismo regulatório, as concentrações séricas fetais, tanto de carotenóides 15,19,51 quanto de retinol, são bastante inferiores quando comparadas às concentrações maternas. 19,51
Segundo Oostenbrug et al.52 os níveis de retinol do RN representam cerca de $55 \%$ do valor sérico materno e os carotenóides cerca apenas 17\%. Resultados semelhantes são descritos por Baydas et al., ${ }^{15}$ Yeum et al. ${ }^{19}$ Dimenstein et al. ${ }^{51}$

Tal condição, no entanto, pode ser agravada em gestantes com DVA. Mesmo que os níveis séricos fetais não sejam gravemente afetados na deficiência subclínica de VA materna, a formação de reservas hepáticas do feto e do recém-nascido pode ser comprometida. 51,53

O transporte desses nutrientes ocorre predominantemente no terceiro trimestre gestacional, 15,22,24 sendo constatada redução dos níveis séricos maternos de retinol e $\beta$-caroteno durante esse período. 52 Entretanto, as reservas do $\mathrm{RN}$ podem ser aumentadas durante o aleitamento materno. 50 A concentração de retinol e carotenóides no leite materno varia durante o período de lactação de acordo com o estado de maturação do leite. A fase do colostro é relatada como sendo a de maior concentração de VA. Essa concentração reduz progressivamente atingindo certa estabilidade a partir da fase do leite maduro. Nesse momento a concentração pode chegar a metade dos níveis iniciais.22,54,55 Estudos sugerem que essa maior transferência de VA durante os primeiros dias de vida seja um mecanismo de correção dos baixos níveis séricos, particularmente de carotenóides, ao nascimento. 54 Esses fatos tornam ainda mais preocupante o hábito existente em algumas sociedades de desprezar o colostro até o início da secreção do leite maduro, privando o $\mathrm{RN}$ do leite materno quando ele é particularmente rico em VA.55

O leite materno é a única fonte de VA durante o período neonatal para lactentes em aleitamento materno exclusivo. A capacidade em atingir as necessidades do RN depende da concentração e do volume de leite consumido, ambos influenciados pelo estado nutricional e ingestão alimentar maternos. Lactentes amamentados por mulheres com adequado estado nutricional de VA estão protegidos contra DVA. 40 Contudo, a deficiência na nutriz pode acarretar transferência inadequada para a criança e perpetuação da DVA, 50 com manifestações de carência subclínica ${ }^{55}$ e sinais clínicos já nos seis primeiros meses de vida, 50 e desenvolvimento de queratomalácia antes do primeiro ano. 40

\section{Estresse oxidativo do nascimento}

Dentre os elementos antioxidantes do organismo podem ser enumerados os captadores de radicais livres (glutationa, vitaminas C e E e carotenóides), as enzimas celulares e extracelulares (superóxido 
desmutase, catalase, glutationa redutase e peroxidase), além da albumina sérica e produtos do metabolismo, como o ácido úrico e a bilirrubina. 26,56,57

A capacidade antioxidante total do plasma juntamente com as enzimas antioxidantes celulares ao nascimento irão determinar como o RN irá resistir ao estresse do nascimento. 57

A hipóxia na vida fetal pode ser uma das causas para a produção de radicais livres e danos oxidativos. ${ }^{23}$ As mudanças na proporção de ácidos graxos poliinsaturados, os quais são bastante susceptíveis à oxidação, constitui outro possível fator envolvido na alteração do status antioxidante do RN. Durante a gestação uma maior mobilização de ácidos graxos leva a um aumento de $51 \%$ dos fosfolipídios plasmáticos maternos. O maior incremento é visto com relação aos ácidos graxos saturados e monoinsaturados, havendo também um aumento significante dos ácidos graxos poliinsaturados. A proporção desses últimos é maior no plasma do RN do que no materno. Em contrapartida os níveis de antioxidantes plasmáticos são menores no RN quando comparados aos maternos. 52 Variação genética, estresse oxidativo materno e capacidade antioxidante materna podem também alterar a capacidade antioxidante do sangue do cordão umbilical. 57

O nascimento, por si só, representa um estresse oxidativo para o RN. A transição pós-natal de um ambiente intra-uterino, relativamente pobre em oxigênio $\left(\mathrm{PO}_{2} 20-25 \mathrm{~mm} \mathrm{Hg}\right)$, para o extra-uterino, significativamente mais rico em oxigênio $\left(\mathrm{PO}_{2} 100\right.$ $\mathrm{mm} \mathrm{Hg}$ ), é tóxico, e expõe o $\mathrm{RN}$ ao aumento da produção de radicais livres 15,22,23,25 levando-o a um desequilíbrio do sistema antioxidante. 26

Após o nascimento, o aumento do estresse oxidativo resultará em redução da capacidade antioxidante, levando ao aumento dos produtos da oxidação de lipídios, proteínas e ácidos nucléicos é esperada e resultando em dano tecidual. 57

Os radicais livres têm uma grande variedade de efeitos potencialmente danosos para o DNA, proteínas, carboidratos e lipídios.18 A peroxidação de ácidos graxos poliinsaturados destrói a função normal da membrana e dos receptores celulares. Pela peroxidação, proteínas podem ser desnaturadas e enzimas desativadas e o DNA pode sofrer danos em suas pontes de ligação. 56,57

\section{Estresse oxidativo e antioxidantes em prematuros}

Os radicais livres têm papel central como causa de parto prematuro e o comprometimento fetal que está associado às enfermidades causadas pelo estresse oxidativo, como inflamação intra-uterina ou materna. Foi verificado, em animais, que a inflamação sistêmica materna que causa parto prematuro está associada à formação de radicais livres e ao estresse oxidativo tanto para a mãe quanto para o feto. 25,26

$\mathrm{O}$ aumento da peroxidação lipídica causada pelo aumento de radicais livres ocorre em recém-nascidos pré-termo (RNPT) e é apontada como sendo um dos mecanismos patogênicos para as doenças causadas por radicais livres do oxigênio, incluindo retinopatia da prematuridade, doença pulmonar crônica e enterocolite necrotizante. 56,57 Um grande número de trabalhos tem sido direcionado para o estudo da relação entre estado nutricional de VA e função pulmonar em RNPT e RN de muito baixo peso.27-29 A VA tem papel importante no desenvolvimento pulmonar fetal. As proteínas surfactantes são reguladas por metabólitos de ácido retinóico. Principalmente no final da gestação, quando ocorre a maturação pulmonar, os estoques de retinol são rapidamente depletados, demonstrando elevadas necessidades de retinol para diferenciação celular e produção de surfactante. 22

Em humanos, e na maioria dos animais estudados, as enzimas antioxidantes atingem altas concentrações apenas no final da gestação $51,56,57$ como forma de preparação para um ambiente extra-uterino relativamente rico em oxigênio. 57 Tem sido demonstrado que RNPT também são capazes de produzir suas próprias enzimas antioxidantes quando estão sob estresse. 56 Contudo, em RNPT que possuem baixas concentrações de enzimas antioxidantes, mesmo níveis normais de antioxidantes plasmáticos podem não prevenir tais agravos se o estresse oxidativo for suficientemente grave. 57

Tanto RNPT, quanto os nascidos a termo, apresentam concentrações de retinol sérico cerca de $50 \%$ inferiores aos níveis maternos. Contudo, os RNPT com baixas concentrações de retinol ao nascimento, levam mais tempo para atingir uma concentração sérica normal no primeiro ano de vida, se comparados aos nascidos a termo, quando ambos os grupos são mantidos em aleitamento materno. Da mesma forma, RN de muito baixo peso apresentam maior risco de desenvolverem DVA, pois seus níveis séricos são significativamente inferiores quando comparados a bebês nascidos a termo. 22 Esse mesmo fato também é observado quando comparam-se RNPT com RN a termo. ${ }^{15,29}$ Estudos têm demonstrado uma correlação positiva entre níveis séricos de antioxidantes e idade gestacional e peso ao nascer. ${ }^{25,57}$ Esses achados corroboram os dados reunidos por Zachman 58 em seu artigo de revisão, no qual descreve vários trabalhos que analisaram a reserva hepática de retinol em RN 
falecidos por diversas causas. O autor encontrou menor reserva hepática em RNPT e de baixo peso. Achado semelhante é observado no trabalho de Ramalho et al., 53 no qual é descrito que os RNs de baixo peso apresentam maior prevalência de inadequação sérica de retinol. As reservas hepáticas ao nascimento também são bastante inferiores quando comparadas às concentrações hepáticas durante a infância. 58

Dessa forma os RNPT necessitam de fontes exógenas de vitaminas para formação de reservas adequadas após o nascimento. As enfermidades e disfunções orgânicas, podem, muitas vezes, limitar a suplementação seja por via parenteral ou enteral. ${ }^{24}$ Há controvérsias com relação à dose de retinol a ser administrada, devido à absorção desta vitamina. Ao equipo de infusão por via parenteral, que pode levar à perda de até $75 \%$ da substância igualmente, a fotodegradação do retinol contido no leite materno administrado por cateter, pode resultar em perdas de até 30\%.42,58,59 Essa tem sido uma das justificativas para sugerir a suplementação de gestantes, para assim garantir uma maior reserva ao nascimento. 24

A exposição a níveis elevados de oxigênio nem sempre é apenas fisiológica (mudança de ambiente), podendo ser também de origem iatrogênica. A terapia pós-neonatal com oxigênio (oxigenioterapia) é necessária em algumas situações, sobretudo nos casos de prematuridade, como forma de garantir $\mathrm{PO}_{2}$ arterial dentro dos limites de normalidade. A exposição direta ao oxigênio é tóxica para tecidos como o pulmão25 e o cérebro. Após introdução dessa medida terapêutica foram descobertos vários efeitos da hiperóxia. Essa alta oferta de oxigênio leva à alta produção de radicais livres capazes de lesar diversos órgãos e tecidos. A alternância entre hipóxia e hiperóxia é danosa e deve ser evitada. Durante a ressuscitação de RN em hipóxia deve-se usar o mínimo de oxigênio extra possível, pois a maioria deles pode ser ressuscitada com o ar ambiente. 60

Dessa forma, RNPT são vulneráveis ao estresse oxidativo ${ }^{57}$ não só devido à deficiência de um ou mais componentes do sistema antioxidante 24 mas também devido ao uso terapêutico do oxigênio. ${ }^{23}$ Sendo assim a homeostase oxidativa é requerida para prevenir danos aos componentes celulares . A exposição abrupta do neonato a níveis elevados de oxigênio pode levar à perda dessa homeostase. ${ }^{26}$

\section{Conclusões}

A importância da VA em momentos de intenso crescimento e desenvolvimento, como na gestação e na infância, é hoje indiscutível devido a seu papel não só na formação e diferenciação celular, como também sua atuação no sistema imune na prevenção de infecções. Mais recentemente a VA ganhou grande destaque no meio científico por sua atuação como antioxidante. Em relação a esse último aspecto, os estudos pioneiros relacionavam-se às doenças crônico-degenerativas e hoje se estendem também às outras situações associadas ao estresse oxidativo, como o momento do nascimento. Nesse contexto ganham importância os carotenóides. Antes estudados por sua ação pró-vitamínica, passam a destacarse por sua potente ação antioxidante.

O tratamento da DVA está bem fundamentado com doses máximas seguras e intervalos de aplicação definidos por organismos internacionais. Ainda se encontra, porém, uma lacuna na literatura quanto às recomendações para RNPT e RN de muito baixo peso, que parecem possuir necessidades especiais e são também os mais vulneráveis à DVA e às suas consequiências. A questão dos carotenóides ainda carece de maiores estudos. Sabe-se que sua ação no tratamento da DVA não atinge os resultados da administração da vitamina pré-formada. Como antioxidantes, não foram estabelecidas doses de suplementação para o grupo materno-infantil, pois questiona-se tal intervenção devido aos possíveis efeitos tóxicos de seus metabólitos, cuja produção aumenta em circunstâncias de estresse oxidativo. Em situações de carência sabe-se que os carotenóides são desviados para a produção de retinol, o que os desvia da ação antioxidante.

Dessa forma, a prevenção desponta como a melhor e mais segura forma de intervenção, a qual deve ser procedida por meio de orientação nutricional, visando a um consumo adequado tanto de VA pré-formada, como das formas pró-vitamínicas. Assim, evita-se que os carotenóides sejam desviados da função antioxidante em prol da produção de retinol em estados carenciais, promovendo-se formação de reservas adequadas de antioxidantes tanto maternas quanto do RN. De igual importância torna-se a manutenção do aporte e transferência pósparto, através também do aconselhamento nutricional à nutriz e do incentivo ao aleitamento materno. O acompanhamento nutricional durante o prénatal é, portanto, fundamental, devendo não só os nutricionistas, mas também os demais profissionais de saúde destinarem maior atenção a esta carência nutricional muitas vezes não valorizada.

Nos casos em que se torna necessária a utilização de alimentação artificial, é fundamental a escolha da fórmula que atenda às necessidades do RN, assim como a avaliação das perdas pela infusão, seja 
por via enteral ou parenteral, aliando-se, quando necessária, à suplementação.

Essas estratégias de intervenção, além de

\section{Referências}

1. IOM (Institute of Medicine). Vitamin A. In: Food and Nutrition Board. IOM (Institute of Medicine. Dietary references intake for vitamin $\mathrm{A}$, vitamin $\mathrm{K}$, arsenic, boron, chromium, copper, iodine, iron, manganese, molybdenum, nickel, silicon, vanadium and zinc. Washington (DC): Nacional Academy Press; 2001. p. 82-161.

2. Sommer A. La carencia de vitamina A y sus consecuencias: guía práctica para la detección y el tratamiento. 3. ed. Ginebra: Organización Mundial de la Salud; 1995.

3. Christian P, West JRKP, Khatry SK, Katz J, Shrestha SR, Kimbrough-Pradhan E, Leclerq SC, Pokhrel RP. Night blindness of pregnancy in rural Nepal - nutritional and health risks. Int J Epidemiol 1998; 27: 231-7.

4. Christian P, West JRKP, Khatry SK, Katz J, Leclerq SC, Kimbrough-Pradhan E, Dali SM, Shrestha SR. Vitamin A or $\beta$-carotene supplementation reduces symptoms of illness in pregnant and lactating women. J Nutr 2000; 130: 267582.

5. Christian P, West JRKP, Kathry SK, Katz J, LeClerq SC, Kimbrough-Pradhan E, Katz J, Shrestha SR. maternal night blindness increases risk of mortality in the first six months of the life mong infants in Nepal. J Nutr 2001; 131: 1510-2.

6. Craft NE. Innovative non or minimally-invasive technologies for monitoring health and nutritional status in mothers and young children. J Nutr 2001; 131:1626S-30S.

7. Diniz AS. Combate à deficiência de vitamina A: linhas de ação e perspectivas. Rev Bras Saúde Matern Infant 2001; 1: 31-6.

8. Ramalho RA, Flores H, Saunders C. Hypovitaminosis A: a public health problem in Brazil. Rev Panam Salud Publica 2001; 12: 117-22.

9. Semba RD, Bulterys M, Munyeshuli V. Vitamin A deficiency and T-cell sub-populations in children with meningococcal disease. J Trop Pediatr 1996; 42: 287-90.

10. Blaner WS. Recents advances in understanding the molecular basis of vitamin A action. Newsletter 1998; 2: 3-6.

11. Sarni RS, Kochi C, Ramalho RA, Schoeps DO, Sato K, Matoso LCQ, Ximenes CF, Souza FI, Damiani RM. Vitamina A, nível sérico e ingestão dietética em crianças e adolescentes com déficit estatural de causa não hormonal. Rev Assoc Med Bras 2002; 48: 48-53.

12. Kulier K, Onis M, Gülmezoglu AM, Villar J. Nutritional interventions for the prevention of maternal morbidity. Int J Gynecol Obstet 1998; 63: 231-46.

13. Radhika MS, Bhaskaram P, Balakrishma N, Ramalakshmi BA, Savitha DEVI, Siva Kumar B. Effects of vitamin A deficiency during pregnancy on maternal and child health. Int J Gynecol Obstet 2002; 109: 689-93.

14. Burri BJ. The formation of vitamin A from $\beta$-carotene: good, bad and variable. Sight and life. Newsletter 2001; 2: 3-5. simples e de baixo custo, são ainda capazes de conferir proteção ao binômio mãe-filho contra a DVA e suas conseqüências.
15. Baydas G, Karatas F, Gursu F, Bozkurt HA, Ilhan N, Yasar A, Canatan H. Antioxidant vitamin levels in term and preterm infants and their relation to maternal vitamin status. Arch Med Res 2002; 33: 276-80.

16. Ramalho RA, Accioly E, Silva LM. Doenças cardiovasculares: efeito antixodante das vitaminas A, C e E. Rev Metab Nutr 2003; 7: 6-9.

17. Nagel E, Vilsendor AM, Bartels M, Pichlmayr R. Antioxidative vitamins in prevention of ischemia/reperfusion injury. Int J Vit Nutr Res 1997; 67: 298-306.

18. Mascio P, Murphy ME, Sies H. Antioxidant defense systems: the role of carotenoids, tocopherols and thiols. Am J Clin Nutr 1991; 53: 194S-200S.

19. Yeum KJ, Ferland G, Patry J, Russel RM. Relationship of plasma carotenoids, retinol and tocopherols in mothers and newborn infants. J Am Coll Nutr 1998; 17: 442-7.

20. Russel RM. Physiological and clinical significance of carotenoids. Int J Vit Nutr Res 1998; 68: 349-53.

21. Paiva SAR, Russel RM. $\beta$-caroteno and others carotenoids as antioxidants. J Am Coll Nutr 1999; 18: 426-33.

22. Bohles H. Antioxidative vitamins in prematurely and maturely born infants. Int J Vit Nutr Res 1997; 67: 321-8.

23. Buonocore G, Perrone S, Longini M, Vezzosi P, Marzocchi B, Paffetti P, Bracci R. Oxidative stress in preterm neonates at birth and on the seventh days of life. Pediatr Res 2002; 52: 46-9.

24. Bolisetty S, Naidoo D, Lui K, Koh THHG, Watson D, Whitehall J. Antenatal supplementation of antioxidant vitamins to reduce the oxidative stress at delivery: a pilot study. Early Hum Dev 2002; 67: 47-53.

25. Robles R, Palomino N, Robles A. Oxidative stress in the neonate. Early Hum Dev 2001; 65 [Suppl]: S75-S81.

26. Buhimschi IA, Buhimschi CS, Pupkin M, Weiner CP. Beneficial impact of term labor: nonenzymatic antioxidant reserve in the human fetus. Am J Obstet Gynecol 2003; 189: 181-8.

27. Kennedy KA, Stoll BJ, Ehrenkranz RA, Oh W, Wright LL, Stevenson DK, Lemons JA, Sowell A, Mele L, Tyson JE, Verter J. Vitamin A to prevent bronchopulmonary dysplasia in very-low-birth- weight infants: has the dose been too low? Early Hum Dev 1997; 49: 19-31.

28. Inder TE, Graham PJ, Winterbourn CC, Austin NC, Darlow BA. Plasma vitamin A levels in the very low birthwieght infant - relationship to respiratory outcome. Early Hum Dev 1998; 52: 155-68.

29. Tammela O, Aitola M, Ikonen S. Cord blood concentration of vitamin A in preterm infants. Early Hum Dev 1999; 56: 39-47.

30. IVACG (International Vitamin A Consultative Group). The bioavailability of dietary carotenoids: current concepts. Sight and life. Newsletter 1999; 4: 32-5. 
31. Christian P, West JRKP, Khatry SK, Katz J, Leclerq SC, Kimbrough-Pradhan E, Shrestha SR. Vitamin A or $\beta$ carotene supplementation reduces but does not eliminate maternal night blindness in Nepal. J Nutr 1998; 128: 145863.

32. West JRKP. Extent of vitamin A deficiency among preschool children and women of reprodutive age. J Nutr 2002; 132: 2857S-66S.

33. Duncan B, Canfield L, Barber B, Greivenkamp J, Oriokot FO, Naluyinda F. The night vision threshold test (NVTT): a simple instrument for testing dark adaptation in young children. J Trop Pediatr 2000; 46: 30-5.

34. Shrestha AK, Duncan B, Taren D, Canfield LM, Greivenkamp JE, Shrestha N, Shrestha KK. A new, simple, inexpensive means of testing functional vitamin A status: the night vision threshold test (NVTT). A preliminary fieldtest report. J Trop Pediatr 2000; 46: 352-6.

35. Filteau SM, Tomkins AM. Promoting vitamin A status in low income countries. Lancet 1999; 353: 1458.

36. McLaren DS, Frigg M. Manual de ver y vivir sobre los transtornos por deficiência de vitamina A (VADD). Washington (DC): OPS; 1999.

37. Stoltzfus RJ, Underwood BA. Breast-milk vitamin A as an indicator of the vitamin A status of women and infants. Bull World Health Organ 1995; 73: 703-11.

38. Sapin V, Alexandre MC, Chaïb S, Bournazeau JA, Sauvant P, Borel P, Jacquetin B, Grolier P, Lémery D, Dastugue B, Azais-Braesco V. Effect of vitamin A status at the end of term pregnancy on the saturation of retinol binding protein with retinol. Am J Clin Nutr 2000; 71: 537-43.

39. WHO (Word Health Organiation), CHD (Child Health Development), Immunization Linked Vitamin A Supplementation Study Group. Randomized trial to assess benefits and safety of vitamin A supplementation linked to immunization in early infancy. Lancet 1998; 352: 1257-63.

40. Vinutha B, Metha MN, Shanbag P. Vitamin A status o pregnant women and effect of post partum vitamin A supplementation. Indian Pediatr 2000; 37: 1188-93.

41. Rice LA, Stoltzfus RJ, De Francisco A, Kjolhede CL. Evaluation of serum retinol, the modified-relative-doseresponse ratio, and breast-milk vitamin A as indicators of response to post partum maternal vitamin A supplementation. Am J Clin Nutr 2000; 71: 799-806.

42. Shenai JP, Rush MG, Parker RA, Chytil F. Sequential evaluation of plasma retinol-binding proein response to vitamin A administration in very-low-birth-weigth neonates. Biochem Mol Med 1995; 54: 67-74.

43. Shenai JP. Vitamin A supplementation in very low birth weight neonates: rationale and evidence. Pediatr 1999; 104: 1369-74.

44. Humphrey JH, Rice AL. Vitamin A supplementation of young infants. Lancet 2000; 356: 422-4.
45. IVACG (International Vitamin A Consultive Group). Maternal night blindness: extent an associated risk factors.

Washington (DC); 1997.

46. IVACG (International Vitamin a Consultive Group). Safe doses of vitamin A during pregnancy and lactation.

Washington (DC); 1998.

47. Rodriguez-Amaya DB. Brazil: a bounty of carotenoid source. Sight and life. Newsletter 2002; 4: 3-9.

48. Bast A, Haenen GRMM, Van der Berg R, Van der Berg H. Antioxidant effects of carotenoids. Int J Vit Nutr Res 1998; 68: 399-403.

49. Russel RM. The vitamin A spectrum: from deficiency to toxicity. Am J Clin Nutr 2000; 71: 878-84.

50. Underwood BA. Maternal vitamin A status and its importance in infancy an early childhood. Am J Clin Nutr 1994; 59 [Suppl]: 517S-24S.

51. Dimenstein R, Trugo NMF, Donangelo CM, Trugo LC, Anastácio AS. Effest of subadequate maternal vitamin A status on placental transfer of retinol and beta-carotene to the human fetus. Biol Neonate 1996; 69: 230-4.

52. Oostenbrug GS. Maternal and neonatal plasma antioxidant levels in normal pregnancy, and the relationship with fatty acid unsaturation. Br J Nutr 1998; 80: 67-73.

53. Ramalho RA, Anjos LA, Flores H. Estado nutricional de vitamina A no binômio mãe/recém-nascido em duas maternidades no Rio de Janeiro, Brasil. Arch Latinoam Nutr 1999; 49: 318-21.

54. Gossage CP, Deyhim M, Yamini S, Douglass LW, MoserVeillon PB. Carotenoid composition of human milk during the first month postpartum and the response to -carotene supplementation. Am J Clin Nutr 76: 2002: 193-7.

55. Ross JS, Harvey WJ. Contribution of breast feeding to vitamin A nutrition of infants: a simulation model. Bull World Health Organ 2003; 81: 80-6.

56. Saugstad OD. Therapy in free radical disease in the newborn. Curr Obstet Gynecol 2000; 10: 103-8.

57. Rogers S, Witz G, Anwar M, Hiatt M, Hegyi T. Antioxidant capacity and oxigen radical diseases in the preterm newborn. Arch Pediatr Adolesc Med. 2000; 154: 544-8.

58. Zachman RD. Retinol (vitamin A) and the neonate: special problems of the human premature infant. Am J Clin Nutr 1989; 50: 413-24.

59. Wardle SP, Hughes A, Chen S, Shaw NJ. Randomised controlled trial of oral vitamin A supplementation in preterm infants to prevent chronic lung disease. Arch Dis Child Fetal Neonatal 2001; 84: F9-13.

60. Saugstad OD. Oxygen toxicity in the neonatal period. Acta Pediatr Scand 1990; 79: 881-92. 